\title{
Luxação traumática do bulbo ocular causada por acidente doméstico
}

\section{Traumatic eyeball luxation caused by domestic accident}

Rogério de Almeida Tárcia'

\section{ResUMO}

A luxação do bulbo ocular ocorre quando há seu deslocamento anterior, de tal forma que as pálpebras se fecham espasmodicamente por detrás dele. Diversas causas não traumáticas são relatadas, sendo que, quando provocado por traumas contusos, com grande envolvimento do nervo óptico e da musculatura extrínseca ocular, o prognóstico em geral é desfavorável. Apresentamos um dramático caso de luxação do bulbo ocular em criança após acidente doméstico, destacando seu mecanismo fisiopatológico e apreciando as condutas.

Descritores: Traumatismos oculares; Ferimentos e lesões; Acidentes domésticos; Criança; Relato de casos [Tipo de publicação]

\footnotetext{
'Médico oftalmologista do Hospital de Pronto-Socorro João XXIII da Fundação Hospitalar do Estado de Minas Gerais; Assistente do Núcleo de Oftalmologia Especializada de Belo Horizonte (MG), Brasil.

Trabalho realizado no setor de emergência do Hospital de Pronto-Socorro João XXIII (Fhemig), Belo Horizonte (MG), Brasil. 


\section{INTRODUÇÃO}

$\mathbf{0}$ trauma ocular é responsável por meio milhão de casos de cegueira no mundo, além de um número considerável de perda parcial da visão, segundo a Organização Mundial da Saúde. Dentro deste contexto, os acidentes domésticos, geralmente subnotificados, são a terceira causa dessas lesões, perdendo tão somente para os acidentes de trabalho e os acidentes automobilísticos ${ }^{(1)}$.

Conceitualmente, trauma não é sinônimo de acidente; este último é uma fatalidade, não passível de prevenção. Trauma é um termo médico que significa lesão. Estas lesões, por sua vez, podem ser prevenidas por medidas educacionais e por mudanças culturais, tais como: o uso sistemático dos óculos de proteção no trabalho e do cinto de segurança no trânsito ${ }^{(2)}$. Nos casos de acidentes domésticos, envolvendo crianças, a prevenção de lesões passa por uma vigilância e educação sistemáticas, nem sempre praticadas nos dias atuais.

Este trabalho tem por objetivo relatar um caso dramático de luxação do bulbo ocular em criança, ocorrido em ambiente doméstico, discutindo as diversas causas relatadas na literatura internacional, as condutas tomadas pelos diversos autores e comentar os seus mecanismos fisiopatológicos.

\section{Relato do caso}

Paciente P.H.B.A. de 10 anos de idade, masculino, natural e procedente de Esmeraldas, MG. Familiares relataram que a criança ao brincar de "pique pega-pega" no escuro dentro de casa batera fortemente contra uma porta entreaberta, provavelmente na maçaneta, do tipo comum, mais popular e reta (alavanca). De imediato notaram a "saída do olho" direito para fora da órbita, sem se acompanhar de vômitos nem tampouco desmaio.

Encaminhado ao Hospital de Pronto-Socorro João XXIII, na capital do Estado, onde o exame inicial revelou um paciente pediátrico lúcido, com preservação dos sinais vitais, e com luxação total do bulbo ocular direito, que se encontrava para fora da órbita, lateralizado e ainda parcialmente retido pela inserção do músculo reto lateral (Figuras 1 e 2). O exame de tomografia computadorizada confirmou a luxação, revelando ainda, fratura da parede anterior do seio maxilar e avulsão do nervo óptico ipsilaterais (Figura 3).

O paciente foi submetido à cirurgia sob anestesia geral para reposicionamento do bulbo ocular e realizado blefarorrafia com suturas de contenção com seda 4-0 (Figuras 4 e 5). O reposicionamento do bulbo ocular dentro da órbita foi conseguido após uma cantotomia lateral, com a ajuda de quatro pinças hemostáticas delicadas, firmemente presas junto às inserções musculares e a quatro mãos. A laceração conjuntival foi reparada com fio absorvível 7-0, mas não foi possível a reinserção dos músculos retos, devido à retração dos cotos.

No seguimento ambulatorial, feito em outro Serviço, tive a notícia de que o olho estava preservado, mas já com sinais de pré-atrofia, com três meses de seguimento e sem percepção luminosa.

\section{Discussão}

A luxação do bulbo ocular, termo derivado do latim Luxatio bulbi, ocorre quando há um deslocamento do bulbo anteriormente, de tal forma que as pálpebras se fecham espasmodicamente por detrás dele, por um espasmo do músculo orbicular. Além de ser um fenômeno raro, esta lesão cria uma forte e negativa impressão em quem a presencia. Quando há lacerações do nervo óptico e de músculos extra-oculares, o termo avulsão do bulbo também pode ser usado ${ }^{(3)}$, como no caso em questão.

Os traumatismos são na sua grande maioria contusos, muitas vezes com o envolvimento dos ossos do terço médio da face. Há relatos de acidentes automobilísti$\cos ^{(4.5)}$, ferimentos por projéteis de arma de fogo ${ }^{(6)} \mathrm{e} e \mathrm{em}$ práticas esportivas ${ }^{(7)}$. O arrancamento do bulbo ocular por agressão ${ }^{(8)}$ ou mesmo por auto-mutilação (Edipismo $)$, encontrado em doentes psicóticos ou usuários de drogas $^{(9)}$, são também relatadas.

Existem diversas causas não traumáticas de luxação do bulbo ocular, algumas vezes provocadas voluntariamente como método de exibição, passíveis de reversibilidade espontânea e que não necessariamente estão associadas a estiramentos do nervo óptico. Exemplos: Síndrome de Crouzón ${ }^{(10)}$, Histiocitose $\mathrm{X}^{(1)}$, Síndrome da Frouxidão Palpebral (floppy eyelid syndrome) ${ }^{(12-14)}$, Doença de Hashimoto ${ }^{(12)}$, anomalias venosas orbitárias congênitas ${ }^{(15)}$ e por manipulação palpebral excessiva, em usuários de lente de contato ${ }^{(16)}$.

Nestas causas não traumáticas fica evidente que aspectos predisponentes estão sempre envolvidos, tais como: órbitas rasas, ligamentos naturalmente mais frouxos, septo orbitário muito posterior e anormalidades musculares $^{(3)}$.

As rupturas dos músculos retos - que são potentes retratores do olho - ajudam a promover a saída do bulbo ocular nos casos traumáticos, além das fraturas ósseas cominutivas e o abrupto aumento da pressão intraorbitária.

Se a luxação do bulbo ocular não é acompanhada 


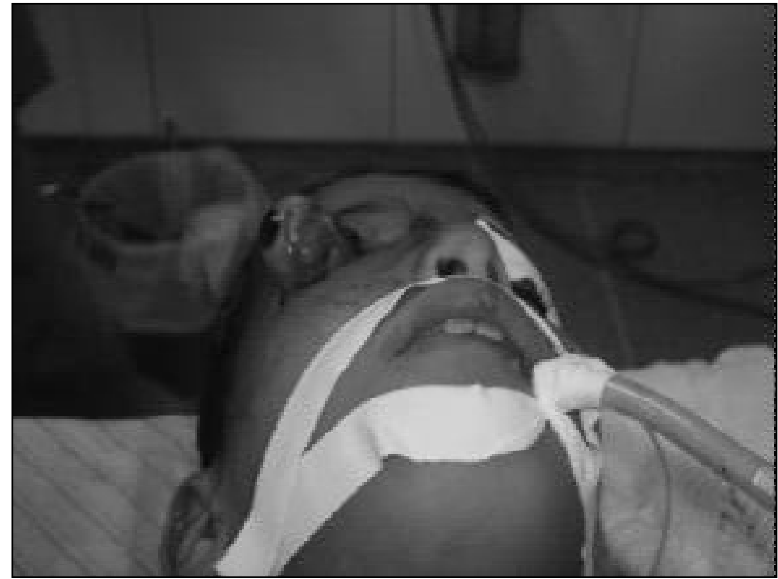

Figura 1: Bulbo ocular direito luxado lateralmente, retido pelo músculo reto lateral. Aspecto pré-operatório, paciente já anestesiado

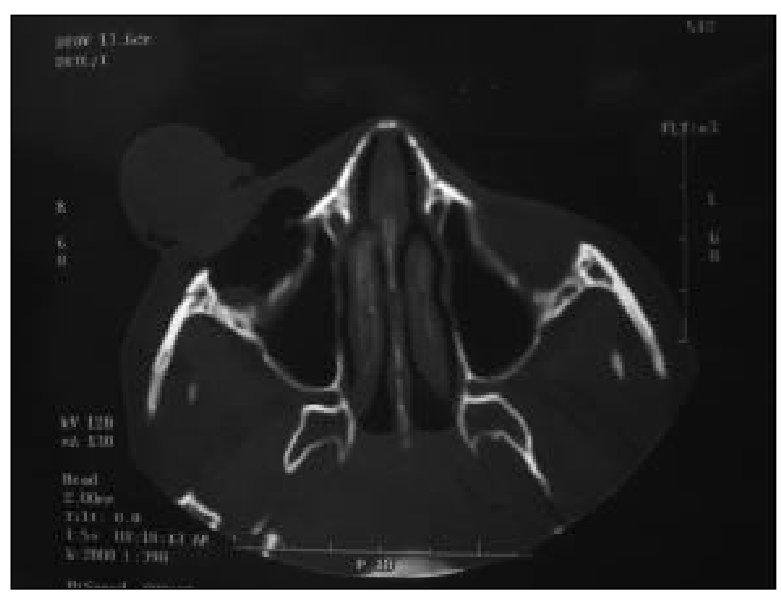

Figura 3: Corte axial de tomografia computadorizada, em "janela óssea". Observa-se a luxação do olho direito e a fratura cominutiva da parede anterior do seio maxilar ipsilateral

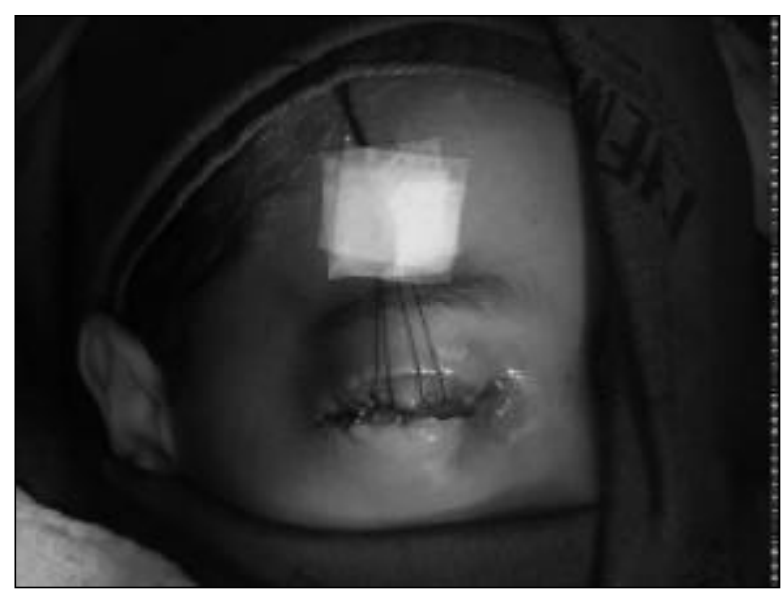

Figura 5: Duas tarsorrafias de contenção com seda 4.0, fixadas junto ao frontal

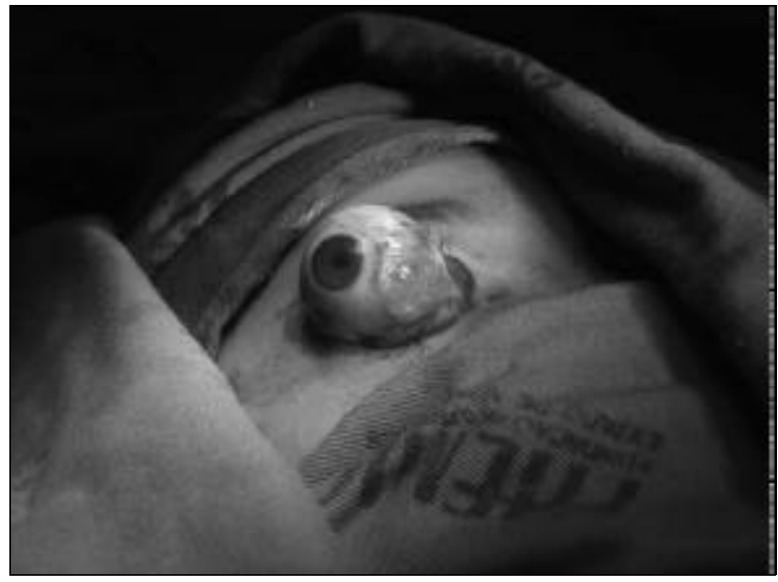

Figura 2: Olho direito luxado lateralmente. Aspecto intra-operatório

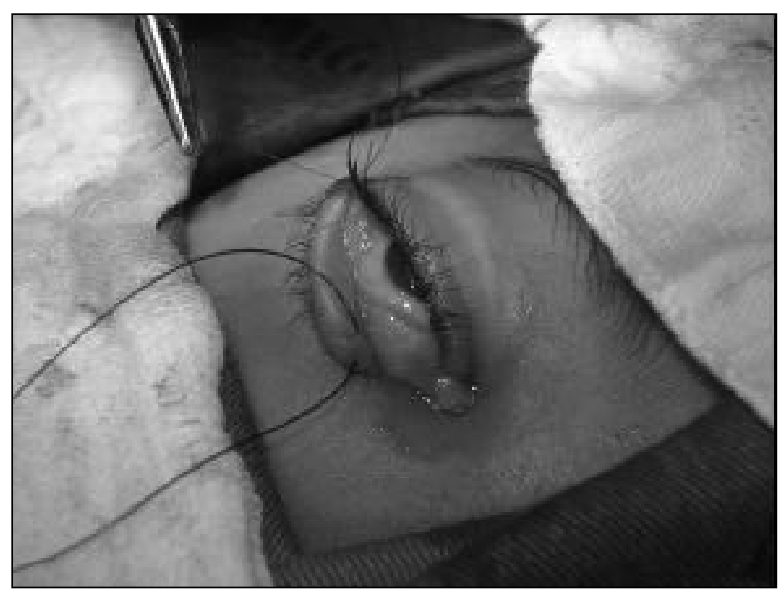

Figura 4: Olho direito já reposicionado na órbita, com fio de náilon 2.0 sondando o canalículo lacrimal inferior lacerado

de avulsão do nervo óptico, a recuperação da visão pode ocorrer. Nesses casos, devemos tentar sob sedação recolocar o olho dentro da órbita, explorando a conjuntiva e não se esquecendo do uso de antiinflamatórios sistêmicos hormonais ou nãohormonais. Exames fundoscópicos subseqüentes são fundamentais no acompanhamento, em busca de hiperemia do disco óptico, palidez e/ou congestão venosa ${ }^{(17)}$. Como mecanismo de proteção do olho, a exemplo de pós-operatório de cirurgias eletivas (ex. catarata) ${ }^{(18)}$, a tarsorrafia lateral pode ser útil em caso de risco de recidiva da luxação.

A grande dúvida está quando a avulsão do bulbo se faz com envolvimento grave do n. óptico e da musculatura extra-ocular. Na literatura se identifica forte tendência em se completar a enucleação, devido ao mau 
prognóstico $^{(6)}$. No entanto, em crianças, a necessidade de se promover um crescimento facial simétrico faz com que a manutenção do bulbo seja importante e devemos pensar em seu reposicionamento ${ }^{(19)}$. Além do ganho psicológico inicial, tanto para o paciente, quanto para a família, uma adaptação de prótese escleral pintada pode ser facilmente experimentada sobre o olho atrófico ${ }^{(20)}$.

\section{Conclusão}

Este relato vem demonstrar que os cuidados com nossas crianças deve ser uma constante preocupação da família e da sociedade, devendo as mesmas receber educação para prevenção de acidentes de forma sistemática. A luxação do bulbo ocular tem diversas causas não traumáticas, sendo que, nos traumas contusos com grande envolvimento do nervo óptico e da musculatura extrínseca ocular, o prognóstico geralmente é desfavorável. O caso em questão corrobora os relatos da literatura quanto a isto.

\section{Abstract}

The eyeball luxation is a dramatic phenomenon in which there is a forward displacement of the eyeball so that the eyelids spasmodically close behind it. There are many risk factors, non-related to trauma, but orbital contusions with optic nerve and extraocular muscles involvement carry the worst prognosis. The present paper reports a dramatic domestic case of globe luxation in childhood, its pathophysiology mechanisms and management.

Keywords: Eye injuries; Wounds and injuries; Domestic, home; Child; Case reports [Publication type]

\section{Agradecimentos}

Ao Dr. Vagner Carvalho Rocha, do Hospital de Pronto-Socorro João XXIII, e ao Dr. Valênio Perez França, do Hospital Mater Dei, pelas preciosas participações na condução do caso.

\section{RefERÊNCIAS}

1. Thylefors B. Epidemiological patterns of ocular trauma. Aust N Z J Ophthalmol. 1992; 20(2): 95-8.

2. Tárcia RA, Lima PN. Anestesia oftalmológica no trauma. In: Moraes RP, editor. Anestesia no trauma. Belo Horizonte: Sociedade de Anestesiologia de Minas ; 2006. p. 199-209.

3. Zengin N, Karakurt A, Gültan E, Kural G. Traumatic globe luxation. Acta Ophthalmol (Copenh). 1992; 70(6): 844-6.

4. Pillai S, Mahmood MA, Limaye SR. Complete evulsion of the globe and optic nerve. Br J Ophthalmol. 1987; 71(1): 69-72.
5. Bajaj MS, Pushker N, Nainiwal SK, Balasubramanya R Traumatic luxation of the globe with optic nerve avulsion. Clin Experiment Ophthalmol. 2003; 31(4): 362-3.

6. Tárcia RA. Avulsão traumática do nervo óptico por projétil de arma de fogo de grosso calibre: relato de caso. Arq Bras Oftalmol. 2006; 69(3): 417-20.

7. Foster BS, March GA, Lucarelli MJ, Samiy N, Lessell S. Optic nerve avulsion. Arch Ophthalmol. 1997; 115(5): 623-30. Erratum in: Arch Ophthalmol. 1997; 115(8):1070.

8. Shneck M, Oshry T, Marcus M, Lifshitz T. Attempted bilateral manual enucleation (gouging) during a physical assault. Ophthalmology. 2003; 110(3): 575-7.

9. Krauss HR, Yee RD, Foos RY. Autoenucleation. Surv Ophthalmol. 1984; 29(3): 179-87.

10. Juárez Echenique JC, Ordaz Favila JC. Luxación espontánea del globo ocular en un caso de síndrome de Crouzón. Rev Mex Oftalmol. 1999; 73(1): 14-6.

11. Wood CM, Pearson AD, Craft AW, Howe JW. Globe luxation in histiocytosis X. Br J Ophthalmol. 1988; 72(8): 631-3.

12. Reyniers R, Paridaens D. Spontaneous globe luxation and floppy eyelid syndrome in a patient with Hashimoto's disease. Eye. 2007; 21(2): 303-4.

13. Apostolopoulos M, Papaspirou A, Damanakis A, Theodossiadis G, Moschos M. Bilateral optic neuropathy associated with voluntary globe luxation and floppy eyelid syndrome. Arch Ophthalmol. 2004; 122(10): 1555-6.

14. Alexandrakis G, Tse DT, Chang WJ. Spontaneous globe luxation associated with floppy eyelid syndrome and shallow orbits. Arch Ophthalmol. 1999; 117(1): 138-9. Comment in: Arch Ophthalmol. 1999; 117(9):1266.

15. Srinivasan S, Gaskell A, McWhinnie H, McClure JP, Corrigan $\mathrm{N}$. Conservative management of globe luxation associated with congenital orbital venous anomaly. J Pediatr Ophthalmol Strabismus. 2003; 40(3): 170-1.

16. Kunesh JC, Katz SE. Spontaneous globe luxation associated with contact lens placement. CLAO J. 2002: 28(1): 2-4.

17. Zorab EC, Burns WL. A case of luxatio bulbi. Br J Ophthalmol. 1940; 24(6): 286-8.

18. Johnson SM, Vestal RY. Lateral tarsorrhaphy for prevention of postoperative complications resulting from globe luxation. J Cataract Refract Surg. 2003; 29(9):1831-3.

19. Vogt P, Motsch S, Mühlendyck H, Hommerich CP. Management of unilateral traumatic bulb luxation with disruption of the optic nerve. HNO. 2003; 51(2): 146-50. Id:German.

20. Kiratli H, Tümer B, Bilgiç S. Management of traumatic luxation of the globe. A case report. Acta Ophthalmol Scand. 1999; 77(3): 340-2.

\section{ENDEREÇO PARA CORRESPONDÊNCIA \\ Dr. Rogério de Almeida Tárcia \\ Av. João Pinheiro $146-13^{\circ}$ andar \\ CEP 30130-922. Belo Horizonte - MG \\ Telefax: (31) 3273-8116 \\ Email: almeidatarcia@yahoo.com.br}

